\title{
The Electromagnetic Form Factor of the Kaon in the Light-Front Approach
}

\author{
Edson O. da Silva, J. P. B. C. de Melo, \\ Victo S. Filho and Bruno El-Bennich \\ Laboratório de Física Teórica e Computacional, LFTC, Universidade Cruzeiro do Sul, \\ 01506-000, São Paulo, Brazil
}

\begin{abstract}
The kaon electromagnetic form factor is calculated within a light-front constituent quark model (LFCQM). The electromagnetic components of the current are extracted from the Feynman triangle diagram within the light-front approach. We also obtain the electroweak decay constant and the charge radius for the kaon in the light-front approach. In this work, the kaon observables are calculated and a fairly good agreement is obtained with a very higher accuracy when compared with the experimental data.
\end{abstract}

Keywords: Light-Front, QCD, quark model, electromagnetic current, electromagnetic form factor, kaon

PACS: $13.40 . \mathrm{Hq}, 14.40 . \mathrm{Be}, 13.40 . \mathrm{Gp}$

With the light-front constituent quark model, $L F C Q M$, it is possible to describe hadronic physics, in terms of $Q C D$ freedom degrees [1]. In this work, we describe in a consistent way the hadronic bound state composite system $q \bar{q}$ (the kaon meson) and the corresponding electromagnetic (e.m.) form factor in the light-front model. In the last years, the pion e.m. form factor has been calculated in many works (for instance, see Refs. [2, 3, 4] and references therein); but for the kaon, there are few theoretical results $[5,6,7]$. The extraction of the electromagnetic form factor in the light-front approach depends on which component of the electromagnetic current (e.c.) is utilized. This is related to problems with the rotational symmetry breaking $[8,9,10,11,12]$. The e.c. with the light-front approach has another contribution, besides the valence contribution to the electromagnetic current. That contribution corresponds to the pair terms added to the matrix elements of the e.c. [8, 10, 13]. In this work, we report results for the kaon e.m. form factor which is extracted from the positive component of the e.c. in the light-front formalism, $J_{K}^{+}=J^{0}+J^{3}$, with a pseudoscalar coupling of the quarks in the Breit frame $\left(q^{+}=0, q_{\perp}=\left(q_{x}, 0\right) \neq 0\right)$. In the case of $J_{K}^{+}$, there is no pair-term contribution in this frame. However, for the $J_{K}^{-}$component of the e.c., the pair term contribution is diferent from zero and necessary in order to preserve the rotational symmetry of the current. Then, the matrix elements of the e.c. within the light-front approach receive other contribution, besides the valence one (see Refs. $[6,9,12]$ and references therein).

Studies concerning light vector and scalar mesons are important because they help our understanding of QCD in the non-perturbative regime.

The kaon e.m. form factor is calculated from the Feynman triangle diagram in the impulse aproximation. The e.c. $J^{\mu}$ can be writen in terms of the charge and quark 
fields $q_{f}$ as:

$$
\begin{aligned}
& J_{q}^{\mu}\left(q^{2}\right)=-\imath 2 e_{q} \frac{m^{2}}{f_{K}^{2}} N_{c} \int \frac{d^{4} k}{(2 \pi)^{4}} \operatorname{Tr}\left[S(k) \gamma^{5} S\left(k-p^{\prime}\right) \gamma^{\mu} S(k-p) \gamma^{5}\right] \Gamma\left(k, p^{\prime}\right) \Gamma(k, p), \\
& J_{\bar{q}}^{\mu}\left(q^{2}\right)=q \leftrightarrow \bar{q} \text { in } J^{\mu}\left(q^{2}\right),
\end{aligned}
$$

in which $N_{c}=3$ is the numbers of colors, $e_{q}$ is the quark (anti-quark) charge and $S(p, m)=1 /\left(p-m^{2}+l \varepsilon\right)$ are the fermion propagators. The calculations are performed in the Breit frame, $\left(q^{+}=0\right)$, with $p^{\mu}=(0,-q / 2,0,0)$ and $p^{\prime \mu}=(0, q / 2,0,0)$, for the initial and final momenta of the system, respectively. The momentum transfer is $q^{\mu}=(0, q, 0,0)$ and $k^{\mu}$ is the spectator quark momentum. The factor 2 appears due to the isospin algebra. The function $\Gamma(k, p)$ is the regulator vertex function, used in order to regularize the Feynman triangle diagram of Eq.(1). We have used as $q \bar{q}$ vertex function the nonsymmetric vertex $\Gamma^{N S Y}(k, p)=N /\left((p-k)^{2}-m_{R}^{2}+\imath \varepsilon\right)$ [9]. The matrix elements of the e.c. for the kaon yields the e.m. form factor

$$
\left(p+p^{\prime}\right)^{\mu} F_{K}\left(Q^{2}\right)=<K\left(p^{\prime}\right)\left|J^{\mu}\right| K(p)>,
$$

where $K$ is the kaon field operator and $Q^{2}=-q^{2}$. The covariant kaon form factor is obtained from Eqs. (1) and (2):

$$
F_{K}\left(q^{2}\right)=-\frac{i 2 e N_{c}}{(2 P)} \frac{m^{2}}{f_{K^{2}}} \int \frac{d^{4} k}{(2 \pi)^{4}} \operatorname{Tr}\left[S(k) \gamma^{5} S\left(P^{\prime}-k\right) \gamma^{\mu} S(P-k) \gamma^{5}\right] \Gamma\left(P^{\prime}, k\right) \Gamma(P, k) .
$$

The $J_{K}^{+}$component of the e.c. is employed to extract the e.m. form factor. The trace in Eq.(3) is the sum of the two contributions

$$
\begin{aligned}
\operatorname{Tr}[1] & =\left[\gamma^{5}\left(\not \not-\not P+m_{u}\right) \gamma^{\mu}\left(\not \not-P /+m_{u}\right) \gamma^{5}\left(\not k+m_{\bar{s}}\right)\right], \\
\operatorname{Tr}[2] & =\left[\gamma^{5}\left(\not \not-\not P+m_{\bar{s}}\right) \gamma^{\mu}\left(\not \not-P /+m_{\bar{s}}\right) \gamma^{5}\left(\not \psi+m_{u}\right)\right] .
\end{aligned}
$$

The complete trace is $\operatorname{Tr}_{\text {kaon }}[\ldots]=(2 / 3) \operatorname{Tr}[1]+(1 / 3) \operatorname{Tr}[2]$, where the factors $2 / 3$ and $1 / 3$ are isospin factors. The integration in Eq.(3) has contributions from two intervals: $0<k^{+}<P^{+}$and $P^{+}<k^{+}<P^{\prime+}$, where $P^{\prime+}=P^{+}+\delta^{+}$. The first interval is the contribution of the valence wave function to the e.m. form factor and the second interval corresponds to the pair-term contribution $[8,9,10,13,14]$. In the case of the nonsymmetric vertex with the plus component of the e.c., the second interval does not contribute at all $[6,9]$. One can verify that only the on-shell pole $\bar{k}^{-}=\frac{f_{1}-l \varepsilon}{k^{+}}$contributes to the $k^{-}$ integration in the interval $0<k^{+}<P^{+}$. Hence, after Cauchy integration in the light-front energy $k^{-}$, Eq. (3) becomes:

$$
\begin{aligned}
F_{\bar{q}}^{+}\left(q^{2}\right)= & e_{q} \frac{N^{2} g^{2} N_{c}}{P^{+}} \int \frac{d^{2} k_{\perp} d x}{2(2 \pi)^{3} x}\left[-4\left(f_{1} x P^{+}-x P^{+} k_{\perp}^{2}-2 f_{1} P^{+}+2 k_{\perp}^{2} P^{+}-\frac{x P^{+} q^{2}}{4}\right)\right. \\
& \left.-\frac{4 f_{1} P^{+}}{x}+8 P^{+}(x-1) m_{q} m_{\bar{q}}-4 x P^{+} m_{q}^{2}\right] \theta(x) \theta(1-x) \Phi_{f}^{*}\left(x, k_{\perp}\right) \Phi_{i}\left(x, k_{\perp}\right) . \\
F_{\bar{q}}^{+}\left(q^{2}\right)= & q \leftrightarrow \bar{q} \text { in } F_{q}^{+}\left(q^{2}\right),
\end{aligned}
$$


where $f_{1}=k_{\perp}^{2}+m_{\bar{q}}^{2}, f_{2}=(P-k)_{\perp}^{2}+m_{q}^{2}, f_{3}=\left(P^{\prime}-k\right)_{\perp}^{2}+m_{q}^{2}, f_{4}=(P-k)_{\perp}^{2}+M_{R}^{2}$ and $f_{5}=\left(P^{\prime}-k\right)_{\perp}^{2}+M_{R}^{2}$. The light-front wave function for the kaon with the nonsymmetric vertex is written like:

$$
\Phi_{q}^{i}\left(x, k_{\perp}\right)=\left[\frac{N}{(1-x)^{2}\left(m_{K^{+}}^{2}-M_{0}^{2}\right)\left(m_{K^{+}}^{2}-M_{R}^{2}\right)}\right],
$$

where $x=k^{+} / P^{+}$is the momentum fraction carried by the quark, $0<x<1$ and $M_{R}^{2}$ is the square mass operator

$$
M_{R}^{2}=M^{2}\left(m_{\bar{q}}, M_{R}\right)=\frac{k_{\perp}^{2}+m_{\bar{q}}^{2}}{x}+\frac{(P-k)_{\perp}^{2}+M_{R}^{2}}{1-x}-P_{\perp}^{2} .
$$

The free quark square mass operator is given by $M_{0}^{2}=M^{2}\left(m_{q}^{2}, m_{\bar{q}}^{2}\right)$. The normalization constant $N$ is determined with the charge conservation condition $F_{K^{+}}^{+}(0)=1$. The calculation of the kaon e.m. form factor in the light-front with $J_{K}^{+}$gives the same result as a covariant calculation [6]. The parameters utilized in the case of the nonsymmetric vertex are the constituent quark masses $m_{q}=m_{u}=0.220 \mathrm{GeV}, m_{\bar{q}}=m_{\bar{s}}=0.508 \mathrm{GeV}$ and the regulator mass $m_{R}=1.0 \mathrm{GeV}$, which are adjusted to fit the charge radius of the kaon. The kaon mass considered in the calculations is the experimental value, $m_{K}=0.496 \mathrm{GeV}[15]$.
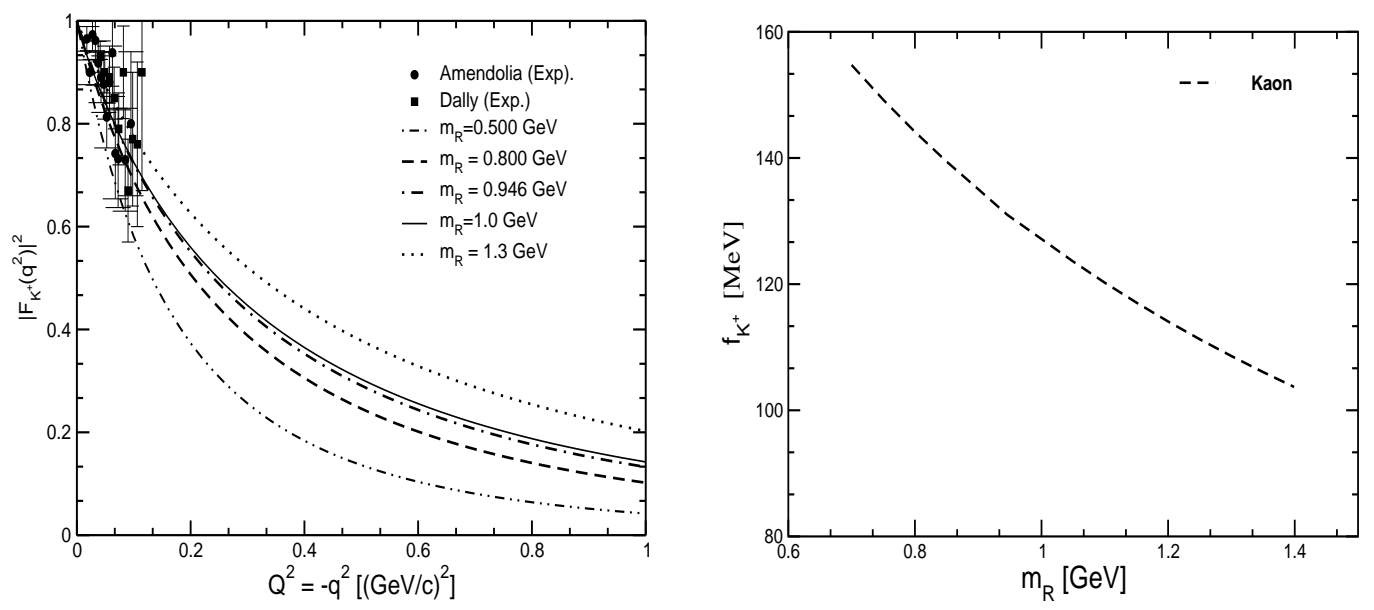

FIGURE 1. The left frame shows the kaon e.m. form factor calculated with the model presented here for different values of $m_{R}$ and compared with the experimental data [16]. The results are covariant and free of the zero modes contributions (see the ref. [2] and references therein). The right frame shows the kaon decay constant for different values of $m_{R}$.

With these parameters, the calculated charge radius of the kaon and the leptonic decay constant are $\left\langle r_{K^{+}}>=0.636 \mathrm{fm}\right.$ and $f_{K^{+}}=126.9 \mathrm{MeV}$, close to the experimental values, $\left\langle r_{K^{+}}>^{\text {exp }}=0.560 \mathrm{fm}\right.$ and $f_{K^{+}}^{\text {exp }}=110.4 \mathrm{MeV}$ [16], and the e.m. form factor for the kaon is shown in the left frame of figure 1. Further, in the right frame of figure 1 , we show the calculated decay constant as function of the regulator mass $m_{R}$. In 
our calculations in such a light-front model, we obtain the same result of a covariant calculation, so that we really verify that $J_{K}^{+}$does not have pair-term contributions for the kaon e.m. form factor. At very low momentum transfer, the light-front model presented here gives better agreement with experimental data $[15,16]$.

Acknowledgments: We thank the brazilian agencies CAPES, Coordenadoria de Aperfeiçomento de Pessoal de Nível Superior, CNPq, Conselho Nacional de Desenvolvimento Científico e Tecnológico, and FAPESP, Fundação de Amparo à Pesquisa do Estado de São Paulo, for financial support.

\section{REFERENCES}

1. S. J. Brodsky, H. C. Pauli and S. S. Pinsky, Phy. Rep. 301, 299 (1998).

2. J. P. B. C. de Melo, T. Frederico, E. Pace, and G. Salmé, Phys. Rev. D73, 074013 (2006).

3. A. F. Krutov, V. E. Troitsky and N. A. Tsirova, Phys. Rev. C80, 055210 (2009).

4. O. Leitner, J.-F. Mathiot and N. A. Tsirova, Eur. Phys. J. A47, 17 (2011).

5. P. Maris and Craig D. Roberts, Phys. Rev. C56, 3369 (1997).

6. Fabiano P. Pereira, J. P. B. C. de Melo, T. Frederico, L. Tomio, Phy. Part. Nucl. 36, 217 (2005).

7. Udit Raha and Hiroaki Kohyama, Phys. Rev. D82 114012 (2010).

8. J. P. B. C. de Melo, J.H.O.Salles, T. Frederico and P. U. Sauer, Nucl. Phys.A631, 574c (1998).

9. J. P. B. C. de Melo, H. W. L. Naus and T. Frederico, Phys. Rev. C59, 2278 (1999).

10. H. W. L. Naus, J. P. B. C. de Melo, and T. Frederico, Few-Body Systems, 24, 99 (1998).

11. J. P. B. C. de Melo and T. Frederico, Phys. Rev. C55, 2043 (1997).

12. B. L. G. Bakker, H.-M. Choi and C. R. Ji, Phys. Rev. D63, 074014 (2001).

13. J. P. B. C. de Melo, T. Frederico, E. Pace, and G. Salmé, Nucl. Phys. A707, 399 (2002). ibid. Braz. J. Phys. Vol. 33, 301 (2003).

14. J. P. B. C. de Melo, T. Frederico, H. W. L. Naus and P. U. Sauer, Nucl. Phys. A660, 219 (1999).

15. K. Nakamura et al. [Particle Data Group Collaboration], J. Phys. G, Nucl. Part. Phys. 37, 075021 (2010).

16. E. B. Dally et al., Phys. Rev. Lett. 45, 232 (1980); S. R. Amendolia et al., Phys. Lett. B178, 435 (1986). 